\title{
Adaptive control of response preparedness in task switching
}

\author{
Marco Steinhauser*, Ronald Hübner, Michel Druey \\ Universität Konstanz, Germany
}

\begin{abstract}
A B S T R A C T
When rapidly switching between two tasks, bivalent stimuli can accidentally trigger the previously executed and therefore still activated response. Recently, it has been suggested that behavioral responserepetition effects reflect response inhibition that reduces the risk of such erroneous response repetitions. The present study investigated neural correlates of this inhibition process using lateralized readiness potentials (LRP). In three experiments, we demonstrate a response-switch bias emerging during the preparatory interval which is independent of task sequence (Experiment 1), which is linked to task preparation (Experiment 2), and which is present only under task-switching conditions (Experiment 3 ). These results suggest that the bias reflects a control process that adaptively regulates response preparedness.
\end{abstract}

Complex human action often consists of a series of elementary tasks. Because these tasks have usually to be executed in a specific order, control processes are necessary which prepare the mental system for every new task. Numerous processes have been identified that support this task preparation, like memory retrieval (Mayr \& Kliegl, 2000), attention adjustment (Meiran \& Marciano, 2002) or cue processing (Koch, 2003). An essential role in this context is played by the control of response preparedness. When confronted with a new task, only task-relevant responses should be in a prepared state. Moreover, response preparedness should adapt to the risk by which specific responses could cause an error. Responses which are at risk to be accidentally triggered by available stimuli should be in a less prepared or even inhibited state. For instance, before one enters the supermarket to buy some healthy vegetables, it could be appropriate to prepare "take the carrots" and to inhibit "take the chocolate".

A special case is given when a response was executed recently, and thus, is still in an activated state. Because the risk to be accidentally triggered by an upcoming stimulus is particularly high for such a response, there should be a general tendency to inhibit the previously executed response. This idea receives support from behavioral studies on task switching showing that repeating the response on consecutive trials can be costly under certain conditions. This response-repetition cost has been attributed to the inhibition of the previously executed response (Druey \& Hubner, 2008a, 2008b; Hübner \& Druey, 2008; Steinhauser \& Htibner, 2006). Our aim in the present study was to demonstrate this inhibition in a

* Corresponding author at: Universität Konstanz, Fachbereich Psychologie, Fach D29, D-78457 Konstanz, Germany. Tel.: +497531 884376.

E-mail address: Marco.Steinhauser@uni-konstanz.de (M. Steinhauser). more direct way by measuring physiological correlates of response preparedness using event-related potentials. Before we introduce our method, though, a brief overview over the relevant paradigms and studies is provided.

The task-switching paradigm (Allport, Styles, \& Hsieh, 1994; Rogers \& Monsell, 1995) is frequently used to investigate processes underlying task preparation. In one version of this paradigm, participants perform multiple tasks in a randomized order in which a cue indicates the relevant task on each trial (Meiran, 1996). Most studies on task switching focused on the so-called switch costs which refer to increased response times and error rates on task-switch trials relative to task-repetition trials and which are attributed to task preparation (Rogers \& Monsell, 1995: Steinhauser, Maier, \& Hübner, 2007) as well as to memory effects (Allport et al., 1994; Schuch \& Koch, 2003; Steinhauser \& Hübner, 2006). Another interesting phenomenon, however, is the complex pattern of response-repetition effects, initially reported by Rogers and Monsell (1995). Numerous studies found a response-repetition cost when the task switched, but a smaller cost or even a response-repetition benefit when the task repeated (e.g., Hübner \& Druey, 2006; Kleinsorge, 1999; Lien, Schweickert, \& Proctor, 2003; Rogers \& Monsell, 1995; Schuch \& Koch, 2004; Steinhauser \& Hubner, 2006).

A variety of theories has been proposed to account for this pattern. Most relevant in the present context is the idea that response-repetition effects in task switching result from the interplay of two processes (e.g., Druey \& Hübner, 2008b; Hubner \& Druey, 2006; Rogers \& Monsell, 1995: Steinhauser \& Hübner, 2006). One process is the inhibition of the previously executed response which should prevent that this response is accidentally triggered by the next stimulus. Because the probability that an accidental response repetition leads to an error is independent of whether the task is repeated or switched, inhibition should be similar for task 
repetitions and task switches. Accordingly, this alone would imply a general response-repetition cost. Usually, however, costs show up only on task-switch trials. This is explained by the assumption that on task-repetition trials, inhibition is compensated by category priming. When the task as well as the response is repeated, priming of the stimulus category has a strong facilitative effect. As a consequence, inhibition produces a response-repetition cost on task-switch trials, whereas category priming reduces this cost or even turns it into a response-repetition benefit on task-repetition trials.

Evidence for this two-process account has been provided, for instance, by Hübner and Druey (2006). In their experiments, stimuli were used which required the same response for each task (congruent stimuli) or a different response for each task (incongruent stimuli). When the previous stimulus was congruent, the responserepetition benefit on task-repetition trials was decreased to the same amount as the response-repetition cost on task-switch trials was increased (see, Fig. 2 in Hübner \& Druey, 2006). In other words, the inhibitory component was selectively increased. To explain this, they argued that congruent stimuli imply stronger activation of the executed response. This, in turn, increases the risk that this response is accidentally triggered by the next stimulus. To counteract this risk, more inhibition of this response is required on the next trial (see also, Druey \& Hübner, 2008b).

A similar increase of the response-repetition cost was shown under conditions of strong time pressure (Steinhauser \& Hübner 2006). From a theoretical view, this is plausible given that time pressure induces a lower response criterion. Under these conditions more inhibition of the previously executed response is beneficial because a low criterion increases the risk that an accidentally triggered response exceeds this criterion. Finally, when stimuli were used that were linked to one task only, inhibition seemed to be largely absent (Hübner \& Druey, 2006). This supports the idea that inhibition is linked to the risk that an error occurs when the stimulus triggers the previously executed response. When stimuli are linked to only one task, then the stimulus is associated only with the correct response and thus cannot trigger the wrong response.

Despite these findings, the behavioral response-repetition effect is rather limited as an indicator of response preparedness. As mentioned above, only a portion of the response-repetition effect can be due to inhibition because the effect of inhibition is blurred by category priming when the task is repeated (e.g., Hubner \& Druey, 2006). Moreover, other authors have proposed mechanisms which might further contribute to the complex response-repetition effects, and which would affect task-repetition trials as well as task switch-trials. For instance, Kleinsorge (1999) suggested that preparing a task-switch generally implies preparation of a response switch and vice versa. Finally, other authors assumed a process called response recoding which alone would imply response-repetition benefits on task-repetition trials but response-repetition costs on task-switch trials (Meiran, 2000; Schuch \& Koch, 2004). Taken together, without precise knowledge of how these mechanisms contribute to the overall pattern, a valid estimation of the inhibitory component of the response-repetition effect is difficult. Because of this, it would be desirable to have a more direct measure of response inhibition.

We assumed that response inhibition can be measured more directly by considering an event-related potential called the lateralized readiness potential (LRP, de Jong, Wierda, Mulder, \& Mulder, 1988; Gratton, Coles, Sirevaag, Eriksen, \& Donchin, 1988). The LRP corresponds to the relative increase of scalp potentials over motor areas contralateral to the response hand, and is regarded as a correlate of the preparedness for a specific hand response. In the context of task switching. LRPs were used to investigate the source of the switch cost and the mechanisms underlying task preparation (de
Jong, Gladwin, \& t Hart, 2006; Gladwin, Lindsen, \& de Jong, 2006; Hsieh \& Liu, 2005; Hsieh \& Yu, 2003a, 2003b).

In the present study, we investigated response inhibition using the LRP as a measure of response preparedness. To this end, a simple task-switching paradigm was applied in which the participants classified digits as odd/even or as less/greater than 5 by responding with their left or right hand, respectively. Task order was randomized and the relevant task on each trial was indicated by a cue preceding the stimulus. The participants were instructed to respond very quickly because time pressure seems to promote the observation of response inhibition (Steinhauser \& Hubner, 2006). Based on the previous considerations, a simple prediction could be derived. If response inhibition influences response preparedness, the LRP should show a bias toward the previously not executed response emerging during the preparatory interval. In the following, we not only demonstrate the existence of such a response-switch bias, but also provide evidence that this bias is linked to response inhibition as postulated by Hubner and Druey (2006): We show that the response-switch bias is similar for task-switch trials and task-repetition trials (Experiment 1). Moreover, we provide evidence that the bias is related to task preparation (Experiment 2), and occurs only under conditions where the stimuli can accidentally trigger the wrong response (Experiment 3).

\section{Experiment 1}

The first experiment tested the crucial predictions of the response-inhibition account. To this end, we examined LRP epochs comprising the response on trial $n-1$ as well as the response on trial $n$. Specifically, we focused on the cue-stimulus interval (CSI) in which participants prepare the upcoming task. According to our hypothesis, a response-switch bias should emerge during this interval reflecting the inhibition of the previously executed response. At stimulus onset on trial $n$, the LRP should be biased toward the correct response if a response switch is required, whereas it should be biased toward the incorrect response if a response repetition is required. Moreover, this bias should be similar for task-repetition trials and task-switch trials. Any LRP effects related to task sequence should emerge not before stimulus onset.

If we observe a response-switch bias emerging during the CSI then the question arises whether this bias can be attributed to task preparation. To allow for such an interpretation, one has to show that the participants really prepare the task during the CSI, which is not self-evident in a procedure like the present one (cf., Altmann. 2004: Koch, 2001; Steinhauser et al., 2007). To test this, we additionally examined electrophysiological correlates of task preparation. Indeed, a number of studies showed a positivity on parietal channels for task-switch trials relative to task-repetition trials, which has been interpreted as a correlate of endogenous task preparation (Barcelo, Escera, Corral, \& Perianez, 2006; Barcelo, Perianez, \& Knight, 2002; Karayanidis, Coltheart, Michie, \& Murphy, 2003: Miniussi, Marzi, \& Nobre, 2005; Nicholoson, Karayanidis, Poboka, Heathcote, \& Michie, 2005; Nicholson, Karayanidis, Bumak, Poboka, \& Michie, 2006; Nicholson, Karayanidis, Davies, \& Michie, 2006). If we find a similar effect, this would support the assumption that task preparation took place during the CSI.

\subsection{Method}

\subsubsection{Participants}

Sixteen participants ( 12 female, 4 male) between 19 and 27 years of age (mean 22.2) with normal or corrected-to-normal vision participated in the study. Participants were recruited at the Universität Konstanz and were paid $5 € / \mathrm{h}$. The study was conducted in 
accordance with institutional guidelines and informed consent was acquired from all participants.

\subsubsection{Stimuli}

Stimuli were the digits 1-9 (without 5) comprising a width of 1.68 visual angle and a height of $2.24^{\circ}$ visual angle at a viewing distance of about $70 \mathrm{~cm}$. A circle and a square with the same size served as cues. Cues and stimuli were presented on a 21 in. color monitor in white color on a black background.

\subsubsection{Design and procedure}

On each trial, the participants had to apply one of two judgments to a digit presented on the screen. In the parity judgment, the digit had to be classified as odd or even. In the magnitude judgment, it had to be classified as less or greater than five. Responses were given by squeezing one of two hand dynamometers (TSD121C, BIOPAC Systems, Goleta, CA, USA) with the left hand (even, less) or the right hand (odd, greater). Participants held one dynamometer in each hand with both arms hanging loose. Each trial started with the presentation of the cue for $200 \mathrm{~ms}$ followed by a blank screen for $600 \mathrm{~ms}$. The cue-task mapping was counterbalanced across participants. The stimulus was presented for $200 \mathrm{~ms}$ followed by a blank screen. A short tone $(600 \mathrm{~Hz}, 100 \mathrm{~ms})$ was presented when response force in one hand exceeded an individually adjusted force threshold. $1000 \mathrm{~ms}$ after the tone, a new trial started. The order of stimuli, judgments and required responses was randomized although stimulus repetitions on two consecutive trials were omitted. The relevant experimental conditions were task sequence (task repetition, task switch) and response sequence (response repetition, response switch). Stimulus presentation and response registration was controlled using custom $\mathrm{C}++$ routines.

participants worked through 10 test blocks of 64 trials, resulting in a total amount of 640 trials. The test blocks were preceded by two practice blocks with 64 trials each, which were not further analyzed. At the beginning of the experiment, the participants' force threshold was determined separately for each hand. To this end, each participant had to squeeze the dynamometers as strong as possible. $20 \%$ of the resulting maximum force was taken as threshold. Participants were encouraged to respond as fast as possible.

\subsubsection{Electrophysiological recording}

Participants were seated comfortably in a dimly lit, electrically shielded room. The electroencephalogram (EEG) was recorded with $\mathrm{Ag} / \mathrm{AgCl}$ electrodes mounted in a cap (Easycap, Herrsching. Germany) from three electrode sites: $\mathrm{C} 3, \mathrm{C} 4$, and $\mathrm{Pz}$. The right mastoid was recorded as an additional channel. Electrodes were referenced to the left mastoid and off-line re-referenced to the average of both mastoids. Electrode impedances were kept below $5 \mathrm{k} \Omega$. Vertical and horizontal electrooculogram (EOG) was recorded from above and below the left eye and from the outer canthi of both eyes, respectively. EEG and EOG were continuously recorded at a sampling rate of $200 \mathrm{~Hz}$ and a high-pass filter of $0.1 \mathrm{~Hz}$ using Biopac amplifiers (BIOPAC Systems, Goleta, CA, USA). Waveforms were off-line filtered with a low pass filter of $5 \mathrm{~Hz}$ for the LRP analyses and $8 \mathrm{~Hz}$ for the remaining ERPs.

\subsubsection{Data analysis}

To analyze behavioral data, response times were computed as the interval between stimulus onset and the time the response threshold for one hand was exceeded. Trials preceded by errors were excluded from all further analyses. EEG data were analyzed by first extracting segments of $2500 \mathrm{~ms}$ before and $1000 \mathrm{~ms}$ after the onset of the stimulus on each trial $n$. As a consequence, these segments contain the current response $\left(R_{n}\right)$ as well as the preceding response $\left(R_{n-1}\right)$. Due to the constant response-cue interval and cue-stimulus interval, these segments are locked to the stimulus on trial $n$ as well as to the response on trial $n-1$. The average voltage in the $100 \mathrm{~ms}$ preceding stimulus onset of trial $n-1$ served as baseline. Trials contaminated with oculomotor artifacts were rejected on the basis of vertical and horizontal EOG. Lateralized readiness potentials were calculated by applying double subtraction method to data from electrode sites $\mathrm{C} 3$ and $\mathrm{C} 4$ (Eimer, 1998). This implies that the difference potential between $\mathrm{C} 3$ and $\mathrm{C} 4$ for trials with righthand responses is subtracted from the same difference potential for trials with left-hand-responses. The data segments were averaged separately for each experimental condition. All analyses were done using custom routines in MatLab 7.0.4 (The Mathworks, Natic, MA, USA).

\subsection{Results}

\subsubsection{Behavioral dato}

Outliers in the response times were eliminated by excluding trials with response times larger than three standard deviations above the mean within each condition of each participant. Response times of correct responses and error rates were subjected to a two-way ANOVA with repeated measurement on the variables task sequence ( $t a s_{k}$ repetition, task switch) and response sequence (response repetition, response switch). In this and the following analyses, main effects are not reported when interactions involving this variable reached significance. The results are depicted in Fig. 1. For the response times, only the main effect of task sequence reached significance, $F(1,15)=7.12, p<0.05$. Response times on task-switch trials were higher ( $542 \mathrm{~ms}$ ) than those on task-repetition trials ( $513 \mathrm{~ms}$ ). For the error rates, the interaction between both variables was significant, $F(1,15)=6.04, p<0.05$. Whereas a response-repetition cost of $6.0 \%$ was obtained on task-

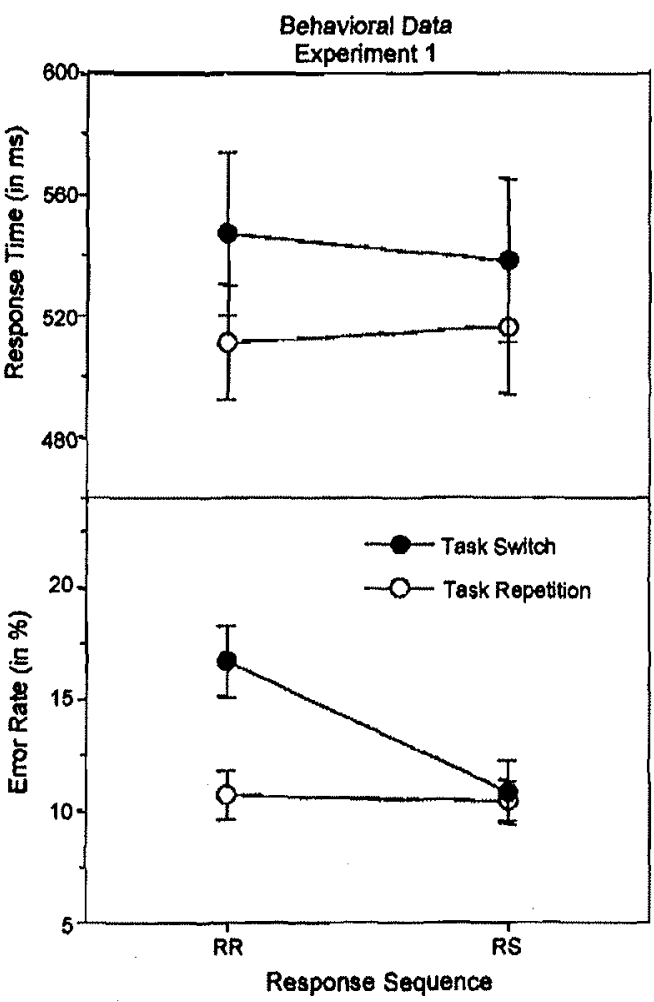

Fig. 1. Experiment 1: response-repetition effects in response times and error rates. 


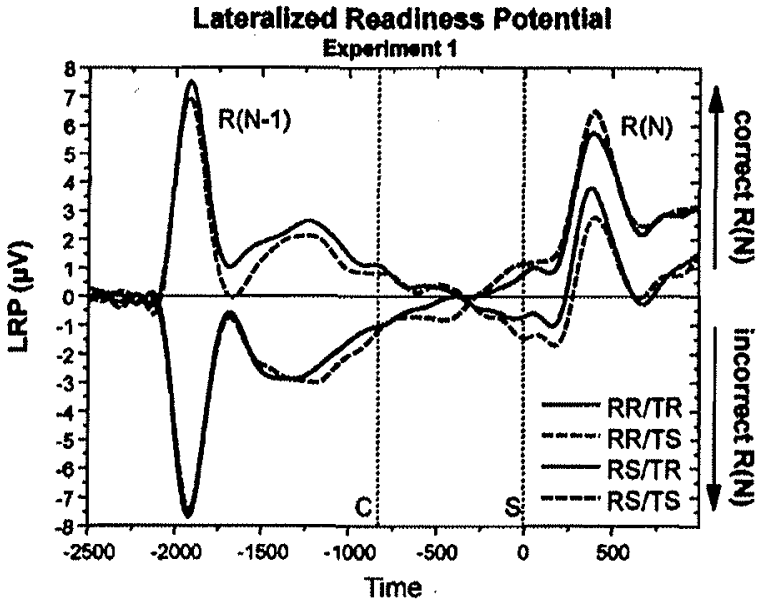

Fig 2. Experiment 1: lateralized readiness potentials (LRP) locked to the stimulus on trial $n$, separately for each combination of task sequence (TR, TS) and response sequence (RR, RS). The range is chosen to comprise the response on trial $n$ as well as that on trial $n-1$. Positive values indicate a higher activation of the response side correct on trial $n, \mu v=$ microvolt, $R(N)=$ response on trial $n, R(N-1)=$ response on trial $n-1 . C=c u e, S=$ stimulus. $R R=$ response repetition, $R S=$ response $s w i t c h$, $T R=$ task repetition, $T S=$ task switch

switch trials, this effect was reduced to $0.3 \%$ on task-repetition trials.

\subsubsection{Lateralized readiness potentials}

Separated LRPs were computed for all combinations of the variables task sequence and response sequence. Fig. 2 shows the resulting waveforms for an interval comprising the responses on trial $n$ and trial $n-1$. Note that the vertical axis is positive for the response side that is correct on trial $n$. Accordingly, the $n-1$ response is represented by a positive deflection on responserepetition trials, whereas the $n-1$ response is represented by a negative deflection on response-switch trials. Further note that the LRP for the $n-1$ response is larger than that for the $n$ response because the LRP for the $n-1$ response is response-locked whereas the LRP for the $n$ response is stimulus-locked. This results because the data segments were averaged locked to the stimulus on trial $n$ as well as to the response on trial $n-1$ (see above).

A closer inspection of this figure allows for two observations: First, during the interval between the $n-1$ response and the stimulus of trial $n$, the LRP develops in a direction contrary to the deflection representing the $n-1$ response. Second, this responseswitch bias seems to be independent of task sequence. Some of the apparent differences between the waveforms reflecting task repetitions and those reflecting task switches seem to exist already before cue onset, and consequently, should be due to noise.

To corroborate these observations statistically, the mean amplitude of the LRP in an interval raging from 100 to $0 \mathrm{~ms}$ preceding stimulus onset was subjected to a two-way ANOVA with repeated measurement on the variables task sequence and response sequence. Only a main effect of response repetition was obtained, $F(1,15)=27.1, p<0.001$, whereas all other effects did not reach significance. Computed as the difference between values for response-switch trials and response-repetition trials, the bias was $1.98 \mu \mathrm{V}$.

In a further step, we examined the question whether the poststimulus LRP related to the $n$ response shows any effects that go beyond those obtained in the pre-stimulus LRP. To ensure comparability of both analyses, we focused on amplitude differences as in the previous analysis. However, now we considered

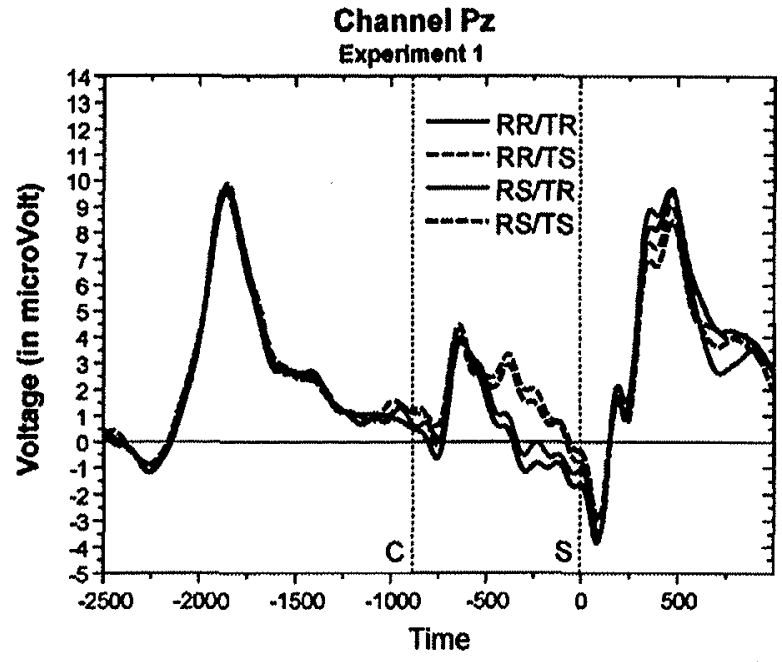

Fig. 3. Experiment 1: stimulus-locked waveforms at electrode Pz, separately for each combination of task sequence (TR, TS) and response sequence (RR, RS). The range is chosen to comprise the response on trial $n$ as well as that on trial $n-1$. range is chosen to comprise the response on trial $n$ as well as that on trial $n-1$. tion, TS m task switch.

the mean amplitude in an interval ranging from 250 to $500 \mathrm{~ms}$ after stimulus onset. Mean amplitudes were subjected to a twoway ANOVA with repeated measurement on the variables task sequence and response sequence. In contrast to the pre-stimulus LRP, we now obtained a significant interaction between both variables, $F(1,15)=5.28, p<0.05$. When the response repeated, higher mean amplitudes were observed for task-repetition trials $(2.72 \mu \mathrm{V})$ than for task-switch trials $(1.71 \mu \mathrm{V})$. In contrast, when the response switched, this difference was reversed (task repetition: $4.78 \mu \mathrm{V}$; task switch: $5.22 \mu \mathrm{V}$ ).

\subsubsection{ERP correlates of task preparation}

To examine correlates of task preparation in the electrophysiological data, we analyzed stimulus-locked waveforms from electrode Pz. Fig. 3 reveals that, during the CSI, waveforms representing task-repetition trials were more positive than waveforms representing task-switch trials. In contrast, response sequence had no substantial effect during this interval. To corroborate these observations, we first calculated the mean amplitude in a prestimulus interval ranging from 500 to $0 \mathrm{~ms}$ before stimulus onset which captures the amplitude of the cue-related slow wave. A one-way ANOVA with repeated measurement on the variable task sequence revealed a significant effect, $F(1,15)=13.7, p<0.01$, indicating an increased mean amplitude on task-switch trials $(1.51 \mu \mathrm{V})$ relative to task-repetition trials $(-0.24 \mu \mathrm{V})$.

\subsection{Discussion}

The first experiment confirmed our predictions. Behavioral effects were mainly obtained for accuracy data, which might be due to the high time pressure. Consistent with earlier results, the error rates revealed a response-repetition cost in case of a task switch, but nearly no cost in case of a task repetition. Most important, however, the electrophysiological data demonstrate the existence of a response-switch bias emerging synchronously with task preparation. During the CSI, the LRP drifted toward the side opposite to the previous response. This resulted in a bias toward the correct response in case of a response switch but a bias toward the incorrect response in case of a response repetition. As predicted, this bias 
was not different for task-repetition trials and task-switch trials Finally, any substantial effects of task sequence emerged after stimulus onset. In the post-stimulus period, the LRP amplitudes largely mirrored the behavioral effect including the interaction between task sequence and response sequence. This supports the inhibition account of the response-repetition effects, according to which these effects are due to (a) an inhibitory effect emerging before stimulus onset and (b) a priming effect emerging after stimulus onset.

In addition, inspection of channel $\mathrm{Pz}$ revealed a significantly more positive waveform during the CSI on task-switch trials, relative to that on task-repetition trials. In earlier studies, similar observations have been attributed to endogenous task preparation (e.g.. Karayanidis et al., 2003). The temporal synchronicity of the LRP bias and the preparatory effect suggests that both effects emerge from the same mechanism. However, because the evidence is only correlational, a further experiment was conducted to investigate the relation between task preparation and the response-switch bias in more detail.

\section{Experiment 2}

The goal of the second experiment was to further examine the process underlying the response-switch bias. The results of the previous experiment were compatible with the idea that the response-switch bias reflects inhibition of the previously executed response during task preparation, and that the function of response inhibition is to prevent that the previously executed response is accidentally triggered again by the next stimulus. Alternatively however, one could argue that the response-switch bias reflects automatic self-inhibition which takes place immediately after response execution. Indeed, such a mechanism has been proposed as a means to prevent perseverative responding ( $\mathrm{Li}$, Lindenberger Ruenger, \& Frensch, 2000; Smith, 1968). Unfortunately, the mere time course of the LRP in the inter-trial interval in the previous experiment cannot distinguish between these alternatives. The fact that the LRP crosses the null line during the CSI does not necessarily imply that inhibition does not already occur before.

One way to distinguish between these alternatives is to examine whether the response-switch bias is linked to task preparation or to the preceding response. Accordingly, we manipulated the interval between the response and the next stimulus (response-stimulus interval, RSI) as well as the interval between the cue and the next stimulus (CSI). Each alternative view makes different predictions as to how the bias is affected by these intervals. If the bias is related to task preparation then it should depend exclusively on the CSI: with a short CSI, task preparation, and thus, the generation of the response-switch bias, cannot occur before stimulus onset. In contrast, if the bias is due to automatic self-inhibition then the RS rather than the CSI should affect the bias. Automatic self-inhibition implies that, after the response on trial $n-1$, the time course of the LRP should be rather ballistic and independent of the time the next cue or the next stimulus arrives. Fig. 1 shows that, $2 \mathrm{~s}$ after the $n-1$ response, the LRP is negative for response switches but positive for response repetitions (which indicates the response-switch bias). In contrast, $1 \mathrm{~s}$ after the $n-1$ response, the LRP is still positive for response switches and negative for response repetitions. Accordingly, if the time course of the LRP is independent of the onset of the next stimulus, then a reduction of the RSI to about $1 \mathrm{~s}$ should imply that a response-repetition bias is obtained.

Based on these considerations, we replicated Experiment 1 with two CSIs $(200,1000 \mathrm{~ms})$ and two RSIs $(1200,2000 \mathrm{~ms}) .^{1}$ Specifi-

\footnotetext{
1 RSi effects in task-switching are typically obtained with smaller RSIs than $1200 / 2000 \mathrm{~ms}$ (e.g. Kach \& Allport, 2006). However, these studies analyzed the influence of RSI on the task-switch costs in order to examine the decay of task sets.
}

cally, we realized a long RSI/long CSI condition, a short RSI/long CSI condition, and a short RSI/short CSI condition. Two contrasts were crucial for our reasoning. The effects of RSI can be examined by comparing the long RSI/long CSI condition with the short RSI/long CSI condition. The effect of CSI can be examined by comparing the short RSI/long CSI condition with the short RSI/short CSI condition. If the response-switch bias is related to automatic self-inhibition, then reducing the RSI should lead to a response-repetition bias (or at least a strongly reduced response-switch bias) at stimulus onset. In contrast, if the response-switch bias is related to inhibition during task preparation, we expect that the response-switch bias at stimulus onset should be eliminated when the CSI is short To obtain a reasonable number of trials per condition despite of the increased number of conditions, we did not include the variable 'task sequence' in the LRP analysis. This seems justified because Experiment 1 showed no significant effect of task sequence on the response-switch bias.

\subsection{Method}

Twelve new participants ( 11 female, 1 male) between 20 and 37 years of age (mean 25.2) with normal or corrected-to-normal vision participated in the study. Stimuli and procedure were the same as in Experiment 1 with the following exceptions. The cue was presented for $150 \mathrm{~ms}$ in all conditions. RSI and CSI were manipulated in three blocked conditions: In condition short RSI/long CSI, an RSI of $1200 \mathrm{~ms}$ and a CSl of $1000 \mathrm{~ms}$ was used. In condition long RSI/long CSI, an RSI of $2000 \mathrm{~ms}$ and a CSI of $1000 \mathrm{~ms}$ was used. In condition short RSI/short CSI, an RSI of $1200 \mathrm{~ms}$ and a CSI of $200 \mathrm{~ms}$ was used. For each condition, two blocks with 128 trials each were performed resulting in a total number of 768 trials. Each participant performed a specific order of the three conditions two times, one time in each session half (resulting in a block sequence like, e.g.. ACBACB). Each of the six possible orders was assigned to two participants. At the beginning of each session, participants performed three practice blocks with 64 trials (one from each condition, in the same order as in the test blocks).

\subsection{Results}

\subsubsection{Behavioral data}

Response time outliers were eliminated by excluding trials with response times larger than three standard deviations above the mean within each condition and each participant. Response times and error rates are depicted in Fig. 4. The effects of CSI and RSI were considered in different analyses.

To examine the effect of $\mathrm{CSI}$, response times of correct responses and error rates were subjected to three-way ANOVAs with repeated measurement on the variables interval (short RSI/short CSI, short RSI/long CSI), task sequence and response sequence. For the response times, we obtained a significant interaction between interval and task sequence, $F(1,11)=20.21, p<0.001$. Task-switch costs were larger in the short RSI/short CSI condition ( $67 \mathrm{~ms}$ ) than in the short RSI/long CSI $(24 \mathrm{~ms})$. For the error rates, the interaction between interval and task sequence was marginally significant $F(1,11)=4.13, p<0.10$, indicating larger task-switch costs in the short RSI/short CSI condition (4.3\%) than in the short RSI/long CSI condition (1.6\%). Moreover, the interaction between task sequence and response sequence reached significance, $F(1,11)=8.60, p<0.05$. A response-repetition cost of $\mathbf{3 . 8 \%}$ was obtained on task-switch

In the present study, we were interested in the influence of RSI on the responseswitch bias. RSI values of 1200 and $2000 \mathrm{~ms}$ were chosen because these values allow for differential predictions derived from the empirical time course of the LRP in Experiment 1. 


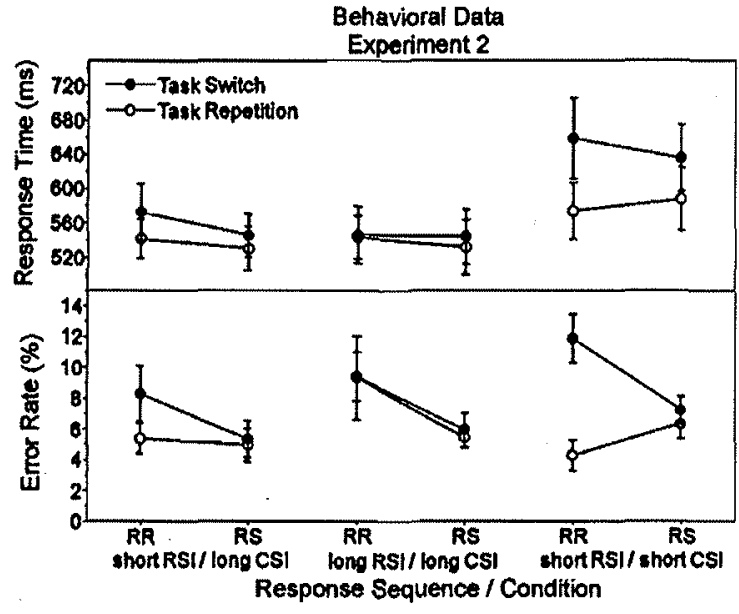

Fig. 4. Experiment 2: response-repetition effects in response times and error rates.

trials, but a small response-repetition benefit was observed on taskrepetition trials $(0.8 \%)$.

To examine the effect of RSI, the same analyses were computed involving the short RSI/long CSI and long RSI/long CSI conditions. For the response times, only a marginally significant effect of task sequence was obtained. $F(1,11)=4.50, p<0.10$, whereas the taskswitch costs were not different for the short RSI/long CSI condition ( $24 \mathrm{~ms}$ ) and the long RSI/long CSI $(8 \mathrm{~ms})$. For the error rates, only a significant main effect of response sequence was obtained, $F(1$, $11)=5.43, p<0.05$, indicating response-repetition costs of $2.6 \%$.

\subsubsection{Lateralized readiness potentials}

Fig. 5 (left column) shows separate LRP waveforms for all combinations of the variables interval and response sequence. The data show a response-switch bias emerging during the responsestimulus interval for the long RSI/long CSI condition and the short $\mathrm{RSI} /$ long CSI condition. Although the bias is somewhat smaller in the short RSI/long CSI condition at stimulus onset, it reaches a similar level in both conditions at the onset of the LRP deflection related to response $n$. For the short RSI/short CSI condition, virtually no response-switch bias is obtained at stimulus onset. However, such a bias seems to develop after stimulus onset.

These observations received support from statistical analyses. Mean LRPs from the interval ranging from 100 to $0 \mathrm{~ms}$ before stimulus onset were extracted. First, to examine the effect of CSI, data from the short RSI/short CSI and short RSI/long CSI conditions were subjected to a two-way ANOVA with repeated measurement on the variables interval and response sequence. We obtained an interaction between interval and response repetition, $F(1,11)=8.18$, $p<0.05$. Whereas a large response-switch bias was obtained in the short RSI/long CSI condition $(2.22 \mu \mathrm{V})$, nearly no effect of response sequence was observed at stimulus onset in the short RSI/short CSI condition $(-0.2 \mu \mathrm{V})$. Second, to examine the effect of RSI, the same analysis was computed involving the short RSI/long CSI and long RSI/long CSI conditions. Here, no significant interaction was obtained, indicating similar response-switch biases in the short $\mathrm{RSI} /$ long CSI condition $(2.22 \mu \mathrm{V})$ and long RSI/long CSI condition $(3.69 \mu \mathrm{V})$.

\subsubsection{ERP correlates of task preparation}

Again, we analyzed stimulus-locked ERPs at electrode Pz separately for task-repetition trials and task-switch trials to examine correlates of task preparation. In this analysis, response sequence was ignored because of the small trial numbers. Moreover, only

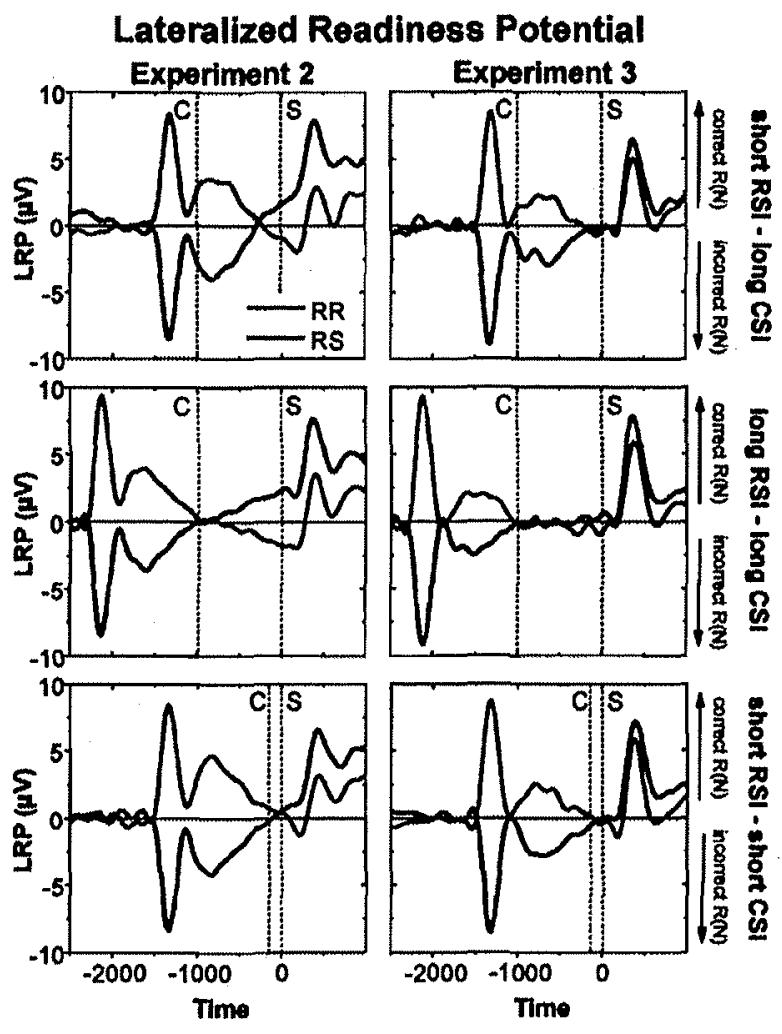

Fig 5. Experiments 2 and 3: lateralized readiness potentials locked to the stimulus on trial $n$, separately for each combination of condition (short RSI/long CSI, long RSI/long CSI, short RSI/short CSI) and response sequence (RR, RS). The tange is chosen to comprise the response on trial $n$ as well as that on trial $n-1$. Positive values indicate a higher activation of the response side correct on trial $n, R(N)=$ response on trial $n, \mu V=$ microvolt. $C$ - cue, $S$ m stimulus, RR - response repetition, $R S$ m response switch, $T R=$ task repetition, TS $=$ task switch.

conditions with long CSI condition were considered because the short CSI is too short for pre-stimulus effects related to task sequence to emerge. Inspection of Fig. 6 revealed a positivity on task-switch trials during the CSI for the short RSI/long CSI condition and the long RSI/long CSI condition. To corroborate this observation, we computed the mean amplitude in an interval ranging from 400 to $600 \mathrm{~ms}$ after cue onset in these two conditions. A two-way ANOVA with repeated-measurement on the variables interval (short RSI/long CSI, long RSI/long CSI) showed only a significant effect of task sequence, $F(1,11)=6.55, p<0.05$, indicating larger mean amplitudes on task-switch trials $(2.93 \mu \mathrm{V})$ than on task-repetition trials $(1.61 \mu \mathrm{V})$.

\subsection{Discussion}

In this experiment we examined whether the response-switch bias reflects response inhibition during task preparation or whether it is due to an automatic self-inhibition of the previous response. Our results clearly favor a preparation-based source of the bias. When the CSI was reduced from $1000 \mathrm{~ms}$ in the short RSI/long CSI condition to $200 \mathrm{~ms}$ in the short RSI/short CSI condition, the bias at stimulus onset nearly disappeared. This suggests that, when preparation cannot occur before stimulus onset, the bias is also not established, which supports the idea that the bias is related to task preparation. 

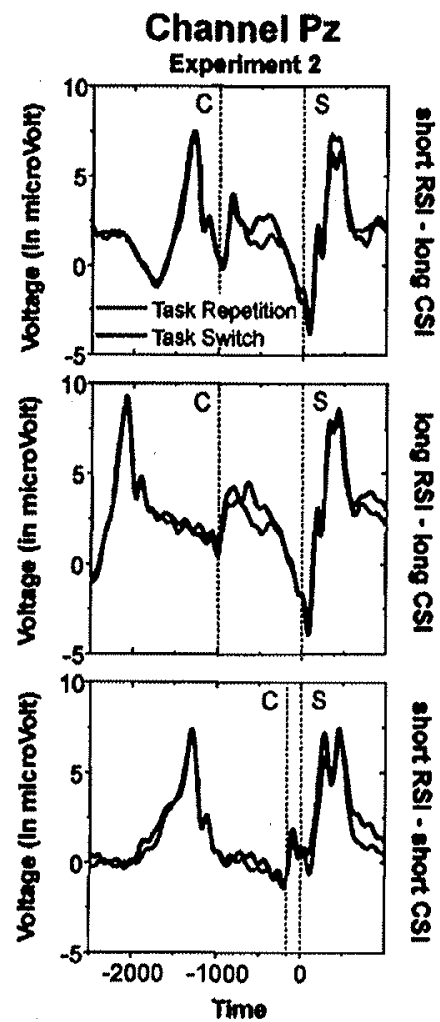

Fig. 6. Experiment 2: stimulus-locked waveforms at electrode $\mathrm{Pz}$, separately for each task sequence (TR, TS), C = cue, $S=$ stimulus, TR m task repetition, TS = task switch.

In contrast, when the RSI was reduced from $2000 \mathrm{~ms}$ in the long RSI/long CSI condition to $1200 \mathrm{~ms}$ in the short RSI/long CS condition, no substantial effect on the response-switch bias was observed. Indeed, there was a trend toward a smaller bias at stimulus onset. However, at the onset of the response-related deflection of the LRP (about $200 \mathrm{~ms}$ after stimulus onset) the bias was nearly identical for the two RSIs. This contradicts the idea of automatic self-inhibition as the source of the response-switch bias. If the bias was due to automatic self-inhibition, the time course of the LRP following the $n-1$ response should be independent of when the next cue or stimulus arrives, and therefore, a different bias should be obtained for a short RSI and for a long RSI. This becomes clear if we compare the time course of the LRP starting with the $n-1$ response in the long RSI/long CSI and short RSI/long CSI conditions (see, Fig. 5, left column). Following the peak associated with the $n-1$ response, the LRP is nearly identical for both conditions until about $1000 \mathrm{~ms}$ after the $n-1$ response. The oscillating form of the LRP during this phase can be attributed to a decay of motor activity and activity from reafferent processes (Leuthold \& Jentzsch, 2001, 2002). After $1000 \mathrm{~ms}$, however, the LRP in the two conditions develops differently. Because task preparation starts earlier relative to the $n-1$ response in the short RSI condition than in the long RSI condition, the response-switch bias is established earlier in the former than in the latter. ${ }^{2}$ This clear shows that the response-switch bias is not

\footnotetext{
${ }^{2}$ Note that the time between cue onset and the onset of the bias is longer in the short RSI condition. This might be due to the fact that with a short RSI, the bias is initially masked by LRP activity linked to the previous response,
}

due to a ballistic self-inhibition or decay process but is rather linked to task preparation during the CSI.

Interestingly, although the response-switch bias at stimulus onset was absent when the CSI was short, inspection of Fig. 5 (left column, last row) suggests that the bias emerges after stimulus onset in this condition. This is consistent with the idea that task preparation is not abandoned with a short CSI, but rather postponed to the post-stimulus period (e.g., Rogers \& Monsell, 1995)..$^{3}$ Based on this observation, one could predict that even in the short RSI/short CSI condition, a response-repetition cost should be observed. Indeed, this expectation is confirmed by our data. In the error rates, the response-repetition costs on task-switch trials were similar in each condition. There was only a trend toward a stronger response-repetition cost on task-repetition trials in the long RSI/long CSI condition. This could reflect a reduced effect of category priming (which normally counteracts the effect of response inhibition on task-repetition trials) which could be due to the fact that the activation of stimulus categories decayed when the RSI was long.

\section{Experiment 3}

The results of the first two experiments demonstrate the existence of a response-switch bias emerging during task preparation. However, these data are not informative with respect to the functional role of this mechanism. Initially, we proposed that the bias prevents that the previously executed response is accidentally triggered by the stimulus. An alternative idea is that the bias simply reflects a tendency to overestimate the probability of response switches. Indeed, studies in which the performance in sequential choice tasks was examined show that, when the interval between two trials is relatively long, participants generate expectancies regarding the probabilities of the involved responses, and thereby often overestimate the probability of a response switch (e.g., Notebaert \& Soetens, 2003; Soetens, 1998). Because this leads to an expectancy bias towards a response switch, the participants prepare for a switch, which leads to costs if the response repeats. Since the inter-trial intervals are usually rather long in taskswitching studies, it seems possible that the response-switch bias is partially due to this expectancy effect.

To test this idea, we conducted a third experiment in which participants performed only one task during the whole experiment. Al other aspects of the procedure, including a cue and a variation of CSI and RSI, were similar as in the previous experiment. Using a constant task not only implies that participants are never confronted with a task switch, it also implies that stimuli are unequivocally associated with one response. This should minimize the risk that the previously executed response is accidentally triggered. If the response-switch bias reflects a mechanism that counteracts this risk, then the bias as well as the response-repetition cost should be eliminated under these conditions. In contrast, if the responseswitch bias simply reflects expectancies, the same bias should be observed.

\subsection{Method}

Twelve new participants ( 8 female, 4 male) between 20 and 32 years of age (mean 22.2) with normal or corrected-to-normal vision participated in the study. Experiment 3 was identical to Experiment 2 with one exception. Participants performed only a single task.

\footnotetext{
3 The fact that task-switch costs (i.e., the performance decrement on task-switch
trials relative to task-repetition trials) are increased with a short $\mathrm{CSI}$ is of ten considtrials relative to task-repetition trials) are increased with a short $\mathrm{CS}$ is often consid-
ered as an indicator that task preparation is postponed to the post-stimulus period (cf., Meiran, 1996; Steinhauser et al., 2007)
} 


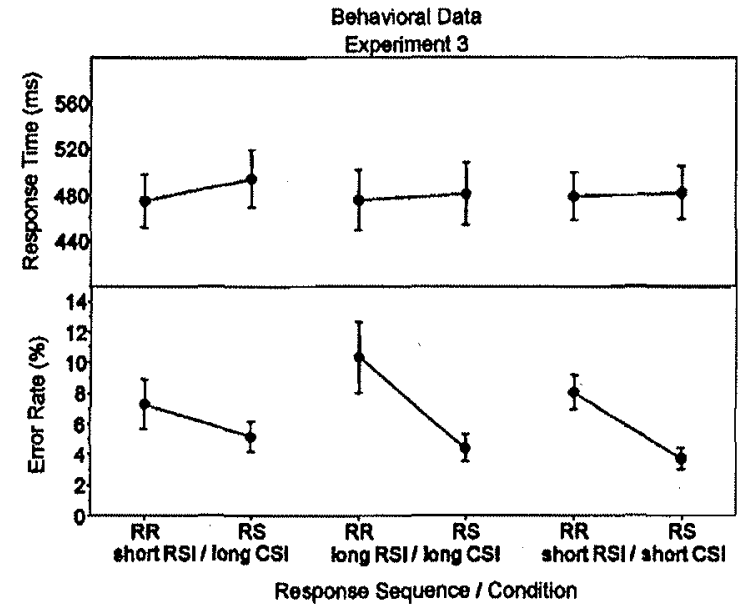

Fig. 7. Experiment 3: response-repetition effects in response times and error rates.

Accordingly, the same cue was presented on each trial. Half of the participants performed the parity task, the other half performed the magnitude task.

\subsection{Results}

\subsubsection{Behavioral data}

Response time outliers were eliminated by excluding trials with response times larger than three standard deviations above the mean within each condition. Response times and error rates are depicted in Fig. 7. Again, the effects of CSI and RSI were considered in different analyses. To examine the effect of CSI, response times of correct responses and error rates were subjected to twoway ANOVAs with repeated measurement on the variables interval (short RSI/short CSI, short RSI/long CSI) and response sequence. For the response times, we obtained only a significant main effect of response sequence, $F(1,11)=5.80, p<0.05$, indicating a response-repetition benefit of $13 \mathrm{~ms}$. For the error rates, response sequence reached significance, $F(1,11)=22.3, p<0.001$, but now a response-repetition cost of $3.2 \%$ was obtained. To examine the effect of RSI, similar analyses were computed involving the short RSI/long CSI and long RSI/long CSI conditions. For the response times, a significant effect of response sequence, $F(1,11)=6.47$, $p<0.05$, indicated a response-repetition benefit of $14 \mathrm{~ms}$. For the error rates, the main effect of response sequence, $F(1,11) \times 9.93$, $p<0.01$, as well as the interaction between response sequence and interval, $F(1,11)=7.71, p<0.05$, were significant. A responserepetition cost was obtained which was larger in the long RSI/long CSI condition (5.9\%) than in the short RSI/long CSI condition (2.1\%).

\subsubsection{Lateralized readiness potentials}

Fig. 5 (right column) shows separate LRP waveforms for all combinations of the variables interval and response sequence. In contrast to Experiment 2 (Fig. 5, left column), we now obtained almost no bias in the pre-stimulus period. The same analyses as in Experiment 2 were conducted but no significant effects were revealed $(F<1)$

\subsection{Discussion}

The results of Experiment 3 partially confirmed our predictions. The response-switch bias disappeared when a constant task was performed. This is consistent with the idea that the role of the bias is to prevent the previously executed and still activated response from being accidentally triggered by the bivalent stimulus. If the stimuli are associated only with the correct response, then there is little risk that the wrong response is triggered. Surprisingly, though, we still obtained a response-repetition cost in the error rates. At first glance, this seems to contradict our reasoning. However, as already discussed, there is evidence for response-repetition costs in serial choice tasks with long inter-trial intervals which reflects participants' expectancy (e.g., Notebaert \& Soetens, 2003; Soetens, 1998). Thus, it is not surprising that we obtained such an effect in the present experiment. But why is such an expectancy-based bias not visible in the LRP?

One answer could be that in the present experiments, expectancy does not lead to a bias in motor activation but to a shift in the motor threshold. That is, when a left-hand response is expected then participants do not increase preparedness of the left hand but rather lower the amount of activation that is necessary for a left-hand response to occur. In this way, the probability that a left response is accidentally elicited is increased. This idea is supported by another observation. In contrast to the previous experiments, the response-repetition cost is observed only in the error rates whereas a small response-repetition benefit occurred in the response times. Such a speed-accuracy tradeoff is a further indicator of a shift in the motor threshold. The fact that such a tradeoff was not observed in our previous experiments suggests that expectancy did not play a role under task switching.

\section{General discussion}

In the present study we investigated the control of response preparedness under task-switching conditions. When preparing a task, it is necessary to minimize the risk that ambivalent stimuli trigger the wrong response. This risk is particularly high for the previously executed response because this response might still be in an activated state. Accordingly, under bivalent stimulus conditions, an efficient task preparation should also include the inhibition of the previously executed resporise. Support for this idea came from studies reporting response-repetition effects in task switching (e.g., Hübner \& Druey, 2006; Rogers \& Monsell. 1995; Steinhauser \& Hübner, 2006). However, this behavioral measure is also affected by other mechanisms like category priming (Hübner \& Druey, 2006), response recoding (Meiran, 2000: Schuch \& Koch, 2004) or expectancy (Notebaert \& Soetens, 2003; Soetens, 1998). Therefore, the present study aimed at providing more direct evidence for response inhibition by examining the LRP, an electrophysiological correlate of response preparedness.

Three experiments were conducted using a standard taskswitching paradigm in which participants alternated randomly between two simple number classification tasks. The behavioral effects replicated the results from earlier studies: responserepetition costs occurred on task-switch trials, whereas no costs or even response-repetition benefits were present on task-repetition trials. In the present study, however, we primarily focused on the analysis of the LRP during task preparation.

In Experiment 1 the participants showed a response-switch bias that emerged during task preparation. This bias can be viewed as reflecting the inhibition of the previously executed response. Moreover, it was similar for task-switch and task-repetition trials, which supports the idea that response inhibition takes place on task-switch trials as well as on task-repetition trials (e.g., Hubner \& Druey, 2008; Hübner \& Druey, 2006). Clearly, from our view this seems reasonable since the risk that the previously executed response is triggered by the stimulus is independent of whether the task repeats or not. Although the bias is similar for both trial types, the behavioral data show a response-repetition cost only on task- 
switch trials. As discussed earlier, this is probably due to the fact that the bias is compensated by priming of stimulus categories (e.g.. "even") which occurs only when the task as well as the response is repeated. Because category priming should emerge during stimulus processing on trial $n$, it cannot affect the response-switch bias during task preparation.

Although the response-switch bias emerged during the CSI, one could argue that the bias is not necessarily related to task preparation because it is possible that participants did not make use of the CSI for preparing the tasks in advance (see, Altmann, 2004; Koch, 2001; Steinhauser et al., 2007). However, the observation of a task-switch positivity on parietal electrode sites which has been attributed to task preparation in earlier studies (e.g., Karayanidis et al., 2003) is not in line which such an assumption.

Nevertheless, we conducted a second experiment, in which we directly examined whether the response-switch bias can be attributed to task preparation or whether it is due to automatic self-inhibition which could occur immediately after the execution of the previous response. To test these hypotheses, we varied the interval between the response and the next stimulus (RSI) as well as the interval between the cue and the next stimulus (CSI). In contrast to the prediction of the self-inhibition account, the time course of the LRP following the $n-1$ response was not independent of RSI. Rather, the bias was established earlier in the short RSI/long CSI condition than in the long RSI/long CSI condition, leading to a similar bias at stimulus onset in the two conditions. However, in line with the preparation account, the bias emerged during the pre-stimulus period only when the CSI was long, and, thus, task preparation could take place during this interval. When the CSI was short, there was a trend toward a bias emerging after stimulus onset. This suggests that task preparation as well as the establishment of a response-switch bias is postponed to the post-stimulus period in this case. This also receives support from the observation that, even with a short CSI, a response-repetition cost on task-switch trials was obtained.

Finally, in a third experiment, we showed that the responseswitch bias disappeared when only a single task had to be performed throughout a block of trials, and, therefore, the stimuli were less likely to trigger the wrong response accidentally. This supports the idea that the bias depends on the risk that the stimulus triggers the wrong response, which is especially high when the stimuli are bivalent, that is, when each stimulus is associated with two tasks which could lead to different responses.

Interestingly, we obtained a pattern of response-repetition effects in this experiment that differed from those in the previous experiments. Whereas a response-repetition cost was observed in the error rates, a response-repetition benefit was present in the response times. We attributed this pattern to expectancy effects which typically emerge when the inter-trial interval is long (e.g. Notebaert \& Soetens, 2003; Soetens, 1998). In our experiment. expectancy could have led to a shift in the motor threshold which would explain the pattern of results. Although such a shift could not to be responsible for the results in Experiments 1 and 2, our result raise the question whether, at least under certain conditions, expectancy effects can contribute to response-repetition costs in task switching

An important issue is how the present results are influenced by the response type used in the present study. In contrast to most task-switching studies, we used squeeze responses. Whereas our behavioral data are very similar to those reported in studies using other response types (e.g., Schuch \& Koch, 2004), the question emerges whether the response-switch bias is related to the squeeze response. In contrast to most other response types, squeeze responses require that participants actively relieve the squeeze by relaxing the grip. Because muscle relaxation has been shown to be associated with various types of motor activity (Buccolieri, Abbruzzese, \& Rothwell, 2004; Pope, Holton, Hassan, Kourtis, \& Praamstra, 2007; Terada, Ikeda, Nagamine, \& Shibasaki, 1995), one could ask whether this could have produced the bias. Basically, such an explanation can be viewed as a variant of the self-inhibition account discussed above. Accordingly, it is contradicted by the finding that the bias is linked to task preparation but not to the response-stimulus interval (Experiment 2 ). If the bias would be induced by relieving the squeeze then it should be independent of whether task preparation is possible during the pre-stimulus period. Rather, it should depend on the time elapsed since the preceding response. Moreover, although muscle relaxation seems to be accompanied by excitatory (Terada et al., 1995) as well as inhibitory cortical activity (Buccolieri et al., 2004), its effect on the LRP has the same direction as that of muscle contraction (Pope et al., 2007). In contrast, the bias in the present study is in the opposite direction as the LRP associated with the previous response. Altogether, it seems highly implausible that muscle relaxation is involved in producing the bias.

Our results demonstrate the advantages of the method used in the present study. The LRP is not only suited to measure response preparedness directly, it is probably an even purer measure than the response-repetition effect in behavioral data. Accordingly, the LRP can now be used to answer further questions regarding the control of response preparedness. One interesting question, for instance, is whether response preparedness is adjusted in an optimal way or whether the risk of errors is overestimated, as suggested by the large number of errors resulting from the response-switch bias itself. Another question is whether the control of response preparedness can be influenced by knowledge about the risk of these errors.

Finally, a particularly interesting question concerns the locus of response inhibition. Although we could measure the effects of response inhibition by considering a correlate of motor preparedness, this does not necessarily imply that response inhibition occurs on the motor level. Rather, it is possible that the control of response preparedness is achieved by activating or inhibiting abstract response codes (like 'left', 'right'). Schuch and Koch (2004) as well as Hubner and Druey (2006) demonstrated that similar behavioral response-repetition effects are obtained if not the actual motor responses are switched or repeated, but rather only the corresponding response codes (e.g., left, right). For instance, if a first task requires pressing left and right keys whereas a second task requires saying 'left' or 'right', then a similar pattern of 'response' repetition effects is obtained. This suggests that the underlying response inhibition affects the activation of semantic response codes, which is then translated into motor preparedness.

Taken together, our results provide evidence for the adaptive control of response preparedness during task preparation. Optimal response preparedness depends not only on which responses are relevant in a given context, but also on which responses are at risk to cause an error. To improve task performance, more "risky" responses are prepared to a lesser extent or are even inhibited. As our results demonstrate, this holds particularly for responses that were recently executed, and, therefore, are at risk to be triggered by the next stimulus.

\section{Acknowledgements}

This research was supported by a grant from the Deutsche Forschungsgemeinschaft (DFG) to the second author (Hu 432/8). We are grateful to Katalin Kermendi and Magdalene Ortmann for assistance in conducting the experiments, and to Iring Koch for valuable comments on an earlier version of this article. 


\section{References}

Allport, A., Styles, E. A., \& Hsieh, S. (1994). Shifting intentional set: Exploring the dynamic control of tasks. In C. Umilta \& M. Moscovitch (Eds.), Attention and performance 15: Conscious and nonconscious information processing (pp. 421-452) Cambridge, MA: The MIT Press.

Altmann, E. M. (2004). Advance preparation in task switching: What work is being done? Psychological Science. 15, 616-622.

Barcelo, F, Escera, C., Corral, M. J., \& Perianez, J. A. (2006). Task switching and novelty

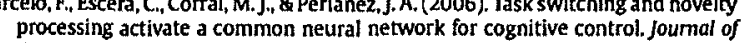
processing activate a common neural
Cognitive Neuroscience, $18,1734-1748$.

Barcelo, F., Perianez, , . A., \& Knight, R. T. (2002). Think differently: A brain orienting response to task novelty. Neuroreport, 13

Buccolieri, A, Abbruzzese, A, \& Rothwell, J. C. (2004). Relaxation from a voluntary contraction is preceded by increased excitability of motor cortical inhibitory circuits. Jourmal of Physiology, 558, 685-695.

de Jong, R, Gladwin, T. E., \& t Hart, B. M. (2006). Movement-related EEG indices of preparation in task switching and motor control. Brain Research, 1105, 73-82.

de Jong, R., Wierda, M., Mulder, G., \& Mulder, L. J. (1988). Use of partial stimulus information In response processing Joumal of Experimental Psychology: Humon Perception and Performance, 14, 682-692.

Druey, M. \& Habner, R (2008a), Effects of stimulus features and instruction on response coding, selection, and inhibition: Evidence from repetition tion on response coding, selection, and inhibition: Evidence from repetition effects under

Druey, M., \& Hübner, R. (2008b). Response inhibition under task switching: its strength depends on the amount of task-irrelevant response activation. Psychological Research, 72, 515-527.

Eimer, M. (1998). The lateralized readiness potential as an on-line measure of central response activation processes. Behavior Research Methods, instruments \& Computers, $30,146-156$.

Gladwin, T. E., Lindsen, J. P., \& de Jong, R. (2006). Pre-stimulus EEG effects related to response speed, task switching and upcoming response hand. Biological Psychology, 72, 15-34

Gratton, G.. Coles, M. C., Sirevaag, E. J., Eriksen, C. W., \& Donchin, E. (1988), Preand poststimulus activation of response channels: $A$ psychophysiological analysis. Joumal of Experimental Psychology: Human Perception \& Performance, 14. ysis. Jour

Hsieh, S., \& Liu, L.-C. (2005). The nature of switch cost: Task set configuration or carry-over effect? Cognitive Brain Research, 22, 165-175.

carry-over effect? Cognitive Brain Research, 22, 165-175,
Hsieh, S., \& Yu, Y.-T. (2003a). Exploring the nature of switch cost: Inferences from

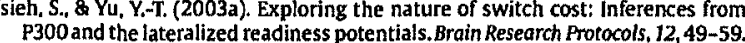
P300 and the lateralized readiness potentials. Brain Research Protocols, 12, 49-59.
sieh, S., \& Yu, Y,-T. (2003b). Switching between simple response-sets: Inferences sieh, S., \& Yu, Y.T. (2003b). Switching between simple response-sets: Inferences
from the lateralized readiness potential. Cognitive Brain Research, 17, 228-237. from the lateralized readiness potential. Cognitive Brain Research, 17, 228-237.
Hubner, R. \& Druey, M. (2008). Multiple response codes play specific roles in response selection and inhibition under task switching. Psychological Research $72,415-424$.

Habner, R., \& Druey, M. D. (2006). Response execution, selection, or activation What is sufficient for response-related repetition effects under task shifting Psychological Research, 70, 245-261.

Karayanidis, F. Coltheart, M., Michie, P. T., \& Murphy, K. (2003). Electrophysiological correlates of anticipatory and poststimulus components of task switching Psychophysiology, 40,329-348.

Kleinsorge, T. (1999), Response repetition benefits and costs. Acta Psychologica, 103 295-310

Koch, 1. (2001). Automatic and intentional activation of task sets. Joumal of Experimental Psychology: Leaming, Memory, and Cognition, 27, 1474-1486.

Koch, I. (2003). The role of external cues for endogenous advance reconfiguration in task switching, Psychonomic Bulletin \& Review, 10,488-492.

Koch, L., \& Allport, A. (2006). Cue-based preparation and stimulus-based priming of casks in task switching. Memory \& Cognition, 34, 433-444.
Leuthold, H., \& Jent zsch, I. (2002), Distinguishing neural sources of movement preparation and execution: An electrophysiological analysis. Biological Psychology, 60, $173-198$

leuthold, $H_{1}$ \& Jentzsch, 1. (2001). Neural correlates of advance movement preparation: A dipole source analysis approach. Cognitive Brain Research, 12, $207-224$

Li, K.Z. H., Lindenberger, U., Ruenger, D., \& Frensch, P. A. (2000). The role of inhibition in the regulation of sequential action. Psychological Science, 11, 343-347.

Lien, M.-C., Schweickert, R, \& Proctor, R. W. (2003). Task switching and response correspondence in the psychological refractory period paradigm. Journal of Experimental Psychology: Human Perception and Performance, 29, 692-712.

Mayr, U, \& Kliegl, R. (2000). Task-set switching and long-term memory retrieval. Joumal of Experimental Psychology: Leaming. Memory, and Cognition. 26, Joumal of

Meiran, N. (1996). Reconfiguration of processing mode prior to task performance. Journal of Experimental Psychology: Learning, Memory, and Cognition, 22. 1423-1442

Meiran. N. (2000). Modeling cognitive control in task-switching. Psychological Research, 63, 234-249.

Meiran. N., Marciano. H. (2002). Limitations in advance task preparation: Switching the relevant stimulus dimension in speeded same-different comparisons. Memory \& Cognition, 30, 540-550

Miniussi, C., Marzi, C. A., \& Nobre, A. C. (2005). Modulation of brain activity by selective task sets observed using event-related potentials. Neuropsychologia, 43. 1514-1528.

Nicholoson, R. Karayanidis, F., Poboka, D. Heathcote, A. \& Michie, P. T. (2005) Electrophysiological correlates of anticipatory task-switching processes. Psychophyslology, 42,540-554.

Nicholson, R., Karayanidis, F., Bumak, E., Poboka, D., \& Michie, P. T. (2006). ERPs

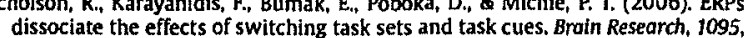
dissociate

Nicholson, R., Karayanidis, F., Davies, A., \& Michie, P. T. (2006). Components of task set reconfiguration: Differential effects of 'switch-to' and 'switch-away' cues. Brain Research, 1121, 160-176.

Notebaert, W. \& Soetens, E. (2003), The influence of irrelevant stimulus changes on stimulus and response repetition effects. Acta Psychologica, 112, 143-156.

Pope, P. A, Holton, A., Hassan, S., Kourtis, D., \& Praamstra, P. (2007). Cortical control of muscle relaxation: A lateralized readiness potential (LRP) investigation. Clinical Neurophysiology, 118, 1044-1052.

Rogers, R. D. \& Monsell, S. (1995). Costs of a predictable switch between simple cognitive tasks. Journal of Experimental Psychology: General, 124, 207-231.

Schuch, S., \& Koch, I. (2003). The tole of response selection for inhibition of task sets in task shifting. Joumal of Experimental Psychology: Human Perception \& Performance, 29, 92-105.

Schuch, S., Koch, I. (2004). The costs of changing the representation of action: Response repetition and response-response compatibility in dual tasks. Joume of Experimental Psychology: Human Perception \& Performance, 30, 566-582.

Smith.M.C.(1968). Repetition effect and short-term memory. Joumal of Experimental Psychology, 77, 435-439.

Soetens, E. (1998). Localizing sequential effects in serial choice reaction time with the information reduction procedure. Joumal of Experimental Psychology: Human Perception and Performance, 24, 547-568.

Steinhauser, M., \& Hubner, R (2006). Response-based strengthening in task shifting: Evidence from shift effects produced by errors. Journal of Experimental Psychology: Human Perception and Performance, 32, 517-534.

Steinhauser, M., Maier, M., \& Hübner, R. (2007). Cognitive control under stress: How stress affects strategies of task-set reconfiguration. Psychological Science, $18,540-545$.

Terada, K, Ikeda, A., Nagamine, T, \& Shibasaki, H. (1995). Movement-related cortical potentials associated with voluntary muscle relaxation, Electroencephalography and Clinical Neurophysiology, 95, 335-345. 